\title{
Rumor detection in social network based on user, content and lexical features
}

\author{
Sushila Shelke ${ }^{1,2}$ (D) Vahida Attar ${ }^{1}$ \\ Received: 21 May 2021 / Revised: 9 August 2021 / Accepted: 21 February 2022 / \\ Published online: 7 March 2022 \\ (C) The Author(s), under exclusive licence to Springer Science+Business Media, LLC, part of Springer Nature 2022
}

\begin{abstract}
Emergence in the social network leads to the extensive and faster diffusion of news than conventional news channels. Verification of data is challenging due to massive information on a social network. Unverified information can be a rumor or fake news that causes damage to an individuals and organizations, revealing the harmful impact on humanity. Therefore, it is vital to combat rumor diffusion to minimize the adverse effects on society. Despite vigorous efforts to deal with this issue, researchers mainly focussed on temporal dynamics of posts and other features like a user, network, content-based, which demonstrate a moderate accuracy. The time series features are associated with an event that suppresses the other quality features related to each post. There is a scope for improvement in the accuracy, so this paper focuses on post-wise features such as user-based, content-based and lexical-based features along with post sequences. We proposed a framework that uses various essential features and combines two deep learning models. Word embedding is utilized with bidirectional long short-term memory (BiLSTM) and combined with post-wise features using a multilayer perceptron (MLP), which improves accuracy. The experiments on the real-world dataset of Twitter demonstrate a notable improvement in accuracy compared to state-of-the-art approaches.
\end{abstract}

Keywords Deep learning $\cdot$ Lexical features $\cdot$ Rumor $\cdot$ Rumor detection $\cdot$ Social network

Sushila Shelke

sss17.comp@coep.ac.in; sushila.shelke@cumminscollege.in

Vahida Attar

vahida.comp@ coep.ac.in

1 Department of Computer Engineering and Information Technology, College of Engineering Pune, Savitribai Phule Pune University, Pune, India

2 Department of Computer Engineering, Cummins College of Engineering for Women, Savitribai Phule Pune University, Pune, India 


$\begin{array}{ll}\text { Abbreviations } \\ \text { BiLSTM } & \text { Bidirectional Long Short-Term Memory } \\ \text { CNN } & \text { Convolutional Neural Network } \\ \text { DL } & \text { Deep Learning } \\ \text { DNN } & \text { Deep Neural Network } \\ \text { GAN } & \text { Generative Adversarial Network } \\ \text { GRU } & \text { Gated Recurrent Unit } \\ \text { LIWC } & \text { Linguistic Inquiry and Word Count } \\ \text { LSTM } & \text { Long Short-Term Memory } \\ \text { ML } & \text { Machine Learning } \\ \text { MLP } & \text { Multilayer Perceptron } \\ \text { PCA } & \text { Principal Component Analysis } \\ \text { RNN } & \text { Recurrent Neural Network } \\ \text { TF-IDF } & \text { Term Frequency-Inverse Document Frequency } \\ \text { UCL } & \text { User-Content-Lexical features }\end{array}$

\section{Introduction}

Exploding various social networking websites (e.g., Twitter, Facebook, Reddit) leads to a high impact on news and information propagation. There are 3.96 billion social media users around the world [36]. However, despite the internet access or the age of a person, $50 \%$ of the globe's population has a routine of using social networks. Stories or news related to politics, economy, social, scientific are uploaded continuously and spread rapidly in social networks, establishing a close real-time view of events or incidences worldwide. Using a social network, the public connected to the world has smooth and faster access to live updates and can help others. Even though it has many advantages, there are challenges verifying the truthfulness of posts [5, 7] and identifying the user who propagates a rumor or fake news on the social network [33]. Original posts are frequently altered by malicious users and disseminated quickly around the internet. Due to these successive moderations, the meaning of the initial post changes in the wrong way. In the rapid diffusion of such news, bots play an important role, where bots are the automatic programs that share fake news with a very high frequency than usual social media users [29]. Fake news and rumor are used alternatively in the literature, but there is a difference in the terms. Fake news is a verifiably false and intentionally misleading article [6]. However, a rumor is a story in circulation, which is unproven at the moment and may get proved as true, false, or unverified at a later stage [43]. The most circulating and misleading stories are based on an individual related to death or defamation, the organization's reputation, the quality of any product, etc.. Such stories are later proven as rumors if those are false. Intentionally spreading such wrong information misled the population and endorsed severe incidences such as violence [39]. Therefore, there is a need for a more accurate and automatic rumor detection system.

Recently, the whole world has been under the fear of coronavirus, which was started in December 2019 and continues to date. There are a few stories related to the COVID vaccine during this pandemic as below.

- COVID-19 Vaccine Cause Herpes [11]

- After the COVID-19 vaccine, blood or plasma donation is not allowed [1]

- COVID-19 killed fewer people than the flu [10] 
Some effective strategies are required to fight the spread of such news, which builds fear and anxiety among society. Many specialty-based fact-checking websites such as Politifact, Snopes, FactCheck [13] work for debunking rumors or fake news. Also, there are crowdsourcing-based fact-checking sites like Twitter [40] and Facebook [12]. The rapid circulation of stories can create chaos within the society if not handled early. In the case of time-dependent events, the consequences can be frightful. News verification through manual efforts is time-consuming. Recognition of rumors and rumor sources [34] can control rumor dissemination.

Many researchers have put forth their views in a rumor detection survey. In [6], they categorize various features broadly into content-based (semantic and lexical features) and context-based (user and network-based) features. They depicted detection in terms of a classification problem by dividing it into four modules, detection, tracking, stance and veracity classification of rumor [43]. Also, reviewers classified the approaches based on machine learning-based (ML) and deep learning-based (DL) techniques. Many researchers have started with an ML-based method to solve the rumor detection problem [18]. However, the manual feature selection in ML approach is tedious and requires physical effort. Therefore, researchers moved on towards the DL-based approach to overcome the problems of ML classification. This research focused on deep learning-based strategies. The review on DL-based methods is depicted in [2], which shows a detailed analysis of the datasets utilized, various deep learning architectures and open challenges in rumor detection. The limitations explained in the existing review involve collecting or selecting benchmarked datasets, size or quality of data, and choice of DL architectures and relevant features.

This paper argues that the crucial focus of previous research was text and temporal features of posts using deep learning. Though few researchers combined time-dependent characteristics with other features such as user, content-based, they use aggregate or fraction values for such features. These aggregate values ignored many essential components associated with an individual post. This research utilizes the significant post-wise features from various categories such as userbased, content-based, lexical and post-based features using different deep learning models.

The contributions of this research are summarized below.

- We have collected a real-world dataset for rumor and non-rumor events from Twitter.

- Identified essential features from different categories such as user, content-based and lexical.

- We have proposed a hybrid deep learning model combining bidirectional LSTM (BiLSTM) and multilayer perceptron (MLP) models.

- We have comprehensively analyzed experimental results on real-world datasets and compared them with state-of-the-art deep learning-based rumor detection approaches.

The current paper has presented a literature review on rumor detection approaches using deep learning frameworks in section 2. The problem definition of rumor detection, data collection and methodology is explained in section 3. Section 4 discuss experimental results and, followed by a conclusion at the end of the paper.

\section{Related work}

The study in this research is a kind of classification problem used in many applications such as Email [30, 31], Sentiment classification [38] and Fake news detection [19]. The research work 
in this paper focused on deep learning-based approaches. According to the different deep learning models used in the current work, we have divided the techniques based on recurrent neural network $(\mathrm{RNN})$, convolutional neural network $(\mathrm{CNN})$ and a combination of different models referred to as Hybrid models.

- RNN based approach: RNN is a form of a feed-forward neural network used to process the sequential data with a variable-length, such as time-series data and is the first to apply for rumor detection by Ma et al. [25]. They extended the basic RNN model with memory unit models like Long Short-Term Memory (LSTM) and Gated Recurrent Unit (GRU), where GRU performs well. The words related to rumor should get more attention, as proposed by Chen et al. [8] using multilayer LSTM and deep attention model. The model depicts the soft attention to the recurrence of distinct features with a specific focus and produces hidden representations in the posts. Guo et al. [17] proposed the same attention-based technique for the hierarchical network of word-post-subevent using bidirectional LSTM. They utilized propagation features such as an average of reposts and comments along with user and post-based features. Chen et al. [9] proposed an unsupervised learning model for rumor detection as an anomaly detection problem on Sina Weibo, a Chinese microblogging website. They utilized microblogs features (like Question mark count, Sentiment score, Pictures count) and posts (like length, count of likes, URL count). The experimental results show that their projected method could attain an accuracy of $92 \%$ and an F1 score of $89 \%$. The self-learning semi-supervised deep learning model and the trust network layer are used in the FakeNewsNet dataset [35], which uses a bidirectional LSTM (BiLSTM) model with a trust layer and shows an F1 score of $88 \%$ [22].

- CNN-based approach: Yu et al. [42] discover that RNN models are not suitable for early detection of rumors with limited input data, so they propose a CNN-based approach for misinformation identification (CAMI). This model can extract essential features from the input sequence and perform well. Rumor identification based on only text features using the BiLSTM-CNN model is projected by Asghar et al. [3], where they proposed a webbased interface for rumor detection. Their method showed an accuracy of $86 \%$. Lin et al. presented a recurrent CNN and combined bidirectional GRU with an attention network, which helps understand the vital information at the word level and learn the temporal features [23]. Also, they utilize the signal words from the text along with a fraction of userbased and content-based features.

- Hybrid Models: Ruchansky et al. [27] proposed a model for fake news detection based on the text of an article, the temporal activity of user response and source users propagating it. They put forward a hybrid model by integrating features from all three categories to get a more precise rumor classification. A recommender system determines a user's genuine interest based on user involvement [32]; therefore, user characteristics are vital. Song et al. [37] proposed a CNN-based model for credible early detection of rumor where they extract feature vectors in each interval using $\mathrm{CNN}$ and feed it to RNN. Also, they suggest that other features related to the user profile and propagation patterns can improve rumor detection. Liu et al. [24] proposed a model for early rumor detection, which combines RNN and CNN to capture temporal patterns of global and local features of users with the propagation paths. They utilized user-related characteristics such as user registration age, geo-enabled, verified users, etc. Data based on information campaign and promoting is used and proposed a Generative Adversarial Network network (GAN) for rumor detection by Ma et al. [26]. Kumar et al. proposed a model in which a sentiment analysis of social 
network users utilized various deep learning models like $\mathrm{CNN}$, a variation of $\mathrm{RNN}$ as long short-term memories (LSTM) with ensemble and attention mechanism [21]. They used Glove (global vector for word representation) for word embedding.

Other than the proposed categories of deep learning-based approaches, the researchers have used ensemble learning for rumor detection and transfer learning for fake news classification. In ensemble learning combination of RNN, GRU and LSTM models is used with various layers in the neural network by Kotteti et al. [20]. A transfer learning using BERT (Bidirectional Encoder Representations from Transformers) model referred to as FakeBERT uses a combination of deep Convolutional Neural Network (CNN) with different kernel sizes and filters by Kaliyar et al. in [19]. The experimental results of the FakeBERT model on the fake news dataset show an excellent accurateness of $98.90 \%$.

In the literature, most of the researchers targeted temporal and text features. While considering temporal features, they think of aggregated features, which hides the importance of post-wise features. Table 1 shows a comparison of deep learning-based methods, where most of the research focuses on textual and temporal characteristics. For text-based features, the most common models are RNN and LSTM. Table 1 depicts the excellent utilization of factors from various categories, which contributes to refining the detection accuracy of rumor and non rumor events. Table 2 compares the performance of benchmarked methods concerning the accuracy and F1 score on a real-world Twitter dataset. This table also presents the text conversion method used in the literature. The most commonly used text representation method in previous work is the term frequency-inverse document frequency (TF-IDF), whereas recently, word2vec is utilized with glove vector. CNN-based methods are mainly used to detect rumors or fake news early. Table 2 reveals that the hybrid model shows excellent performance in terms of accuracy of $89 \%$ [27]. In Table 2, methods used in [8, 27, 37] consider either overall accuracy or cross-validation. From the literature, we can conclude that we have a scope for improvement in the preciseness of rumor detection.

Due to the advantage of the hybrid model from the literature, this research combines the BiLSTM model with Multilayer Perceptron (MLP) as a hybrid deep learning model. It explores different attributes from the user, content-based, lexical and text of a post. Features from each category are listed in section 3 .

Table 1 Deep learning-based approaches with various features

\begin{tabular}{|c|c|c|c|c|c|c|}
\hline \multirow[t]{2}{*}{ Ref. no. } & \multirow[t]{2}{*}{ Approach } & \multirow[t]{2}{*}{ Models } & \multicolumn{4}{|c|}{ Features type } \\
\hline & & & User & Text & Propagation & Temporal \\
\hline$[25]$ & RNN & RNN, LSTM + GRU, Multilayer GRU & & $\sqrt{ }$ & & $\sqrt{ }$ \\
\hline [8] & $\mathrm{RNN}$ & RNN+Multilayer LSTM & & $\sqrt{ }$ & & $\sqrt{ }$ \\
\hline [17] & $\mathrm{RNN}$ & Bi-LSTM & $\sqrt{ }$ & $\sqrt{ }$ & $\sqrt{ }$ & \\
\hline [9] & $\mathrm{RNN}$ & $\mathrm{RNN}+$ Autoencoder & $\sqrt{ }$ & $\sqrt{ }$ & & $\sqrt{ }$ \\
\hline [42] & $\mathrm{CNN}$ & CNN+Max Pooling & & $\sqrt{ }$ & & $\sqrt{ }$ \\
\hline [3] & $\mathrm{CNN}$ & Bi-LSTM & & $\sqrt{ }$ & & \\
\hline [23] & $\mathrm{CNN}$ & $\mathrm{CNN}+\mathrm{Bi}-\mathrm{GRU}$ & $\sqrt{ }$ & $\sqrt{ }$ & & $\sqrt{ }$ \\
\hline [27] & Hybrid & $\mathrm{RNN}+\mathrm{NN}$ & $\sqrt{ }$ & $\sqrt{ }$ & & $\sqrt{ }$ \\
\hline$[37]$ & Hybrid & $\mathrm{RNN}+\mathrm{CNN}$ & & $\sqrt{ }$ & & $\sqrt{ }$ \\
\hline$[24]$ & Hybrid & $\mathrm{GRU}+\mathrm{CNN}$ & $\sqrt{ }$ & & $\sqrt{ }$ & $\sqrt{ }$ \\
\hline$[26]$ & Hybrid & $\mathrm{GAN}+\mathrm{CNN}, \mathrm{GAN}+\mathrm{GRU}$ & & $\sqrt{ }$ & & $\sqrt{ }$ \\
\hline
\end{tabular}


Table 2 Comparative study of performance metrics

\begin{tabular}{lllllll}
\hline Ref. no. & Approach & Text representation & Model & Class & Accuracy & F1 \\
\hline$[25]$ & RNN & tf-idf & GRU2 & R & 0.881 & 0.898 \\
& & & & NR & & 0.86 \\
{$[8]$} & RNN & tf-idf & CallAtRumors-LSTM & - & - & 0.87 \\
{$[17]$} & RNN & tf-idf & HSA-BLSTM & R & 0.844 & 0.825 \\
& & & & NR & & 0.863 \\
{$[42]$} & CNN & Paragraph2vector & CAMI-CNN & R & 0.777 & 0.793 \\
& & & & NR & & 0.758 \\
{$[23]$} & CNN & word2vector & RCNN-FAN & R & 0.799 & 0.792 \\
& & & & NR & & 0.805 \\
{$[27]$} & Hybrid & doc2vec & CSI-LSTM+NN & - & 0.892 & 0.894 \\
{$[37]$} & Hybrid & tf-idf & CED-CNN+RNN & - & 0.744 & 0.747 \\
{$[24]$} & Hybrid & tf-idf & GAN-GRU & R & 0.863 & 0.866 \\
& & & & NR & & 0.858 \\
\hline
\end{tabular}

\section{Methodology}

This section presents problem definition, data collection and pre-processing, feature selection and methodology followed using a deep learning model.

\subsection{Problem definition}

The rumor detection in social networks formally presented as the event-wise sequence of posts given as input to the proposed model identifies whether the event is rumor or non-rumor. The event is any condition or incident that happened around us and informed through news, messages, like news-related bomb blasts, political statements, targeted organizations, etc. The input data contains a set of events $E=\left\{e_{1}, e_{2}, \ldots, e_{N}\right\}$, where $\mathrm{N}$ is the total number of events. Each event includes $\mathrm{n}$ posts, as $e_{1}=\left\{p_{1}, p_{2}, \ldots, p_{n}\right\}$, where $\mathrm{n}$ is varying in size and $\mathrm{p}$ contains message or tweet related to the event. Along with the posts, other features are extracted, such as, UserVect $=\left\{u_{1}, u_{2}, \ldots, u_{n}\right\}$, where $\mathrm{u}_{1} \ldots \mathrm{u}_{\mathrm{n}}$ are user features like user registration age, friend count, etc., ContVect $=\left\{c_{1}, c_{2}, \ldots, c_{\mathrm{n}}\right\}$, where $\mathrm{c}_{1} \ldots \mathrm{c}_{\mathrm{n}}$ are contentbased features like number of question marks, number of URLs, etc. and LexVect $=\left\{l_{1}, l_{2}\right.$, $\left.\ldots, l_{n}\right\}$, where $l_{1} \ldots l_{n}$ are lexical features. The proposed model's goal is to check whether the event is a rumor or not by considering various post-related features.

\subsection{Data set}

This research focused on the Twitter microblogging website. The data collection for rumors involves identifying rumor and non-rumor events from debunking sites, collecting data related to each event from Twitter and finally, cleaning data. This section presents the entire process of data curation.

\subsubsection{Data collection}

We have identified rumor and non-rumor events from debunking sites of www.snopes.com and www.politifact.com. These sites have the details of story circulation, evidence of news, 
statuses such as True or False after investigation and the published date of the story. After verifying each tale with True or False, the events are categorized into Rumor and Non-rumor. Figure 1 shows the recognition of rumor events from snopes.com with a rating as False [16] and Fig. 2 shows the determination of non-rumor events from the Politifact site with news status as "Mostly True" [4]. Figures 1 and 2 present the example of event identification from various websites. The tweets were collected for each event from 1st March 2020 to 31st March 2020. Twitter's 30-day endpoint premium API paid scheme extracts tweets from the last 30 days during the above period. The data for each event is collected by writing different search queries. Figure 3 shows the data collected for news by altering the keywords in search queries highlighted in bold. The sample examples of finalized events related to rumor and nonrumor are listed in Tables 3 and 4. These tables give details of event headline, count of posts for each event and date of data collection.

The statistics for real-world data collected from Twitter are given in Table $5.78 \%$ of events are identified from politifact.com and $22 \%$ are from snopes.com. Besides the dataset we formed, we also utilize the publicly available Twitter dataset [28], constructed by [25] for rumor detection. Due to Twitter's policy, only tweet ids are given in a dataset for rumor and non-rumor events. Therefore, we have extracted tweets for each tweet id in JSON format. This research focuses on English posts only. Twitter does not provide data for a few events for reasons such as user does not exist, user suspended. After data pre-processing, the events having a single post and non-English language are removed from the dataset. Therefore, the final count for the events is 986 , whereas the total posts become 267,708 . The detailed steps of data pre-processing are explained further.

\subsubsection{Data pre-processing}

The data extracted from Twitter contains URLs, hashtags, mentions, emoticons, special characters, etc., which need to be preprocessed to use the cleaned text data as input to our model. The text data is prepared by removing URLs, hashtags, mentions, emoticons, punctuations, and non-ASCII characters using the python regex library represented by re. The duplicate posts are removed from the dataset. We have expanded the contractions

\section{Claim}

Gargling with salt water or Vinegar 'eliminate' the COVID-19 coronavirus from the throat of an infected person's system.

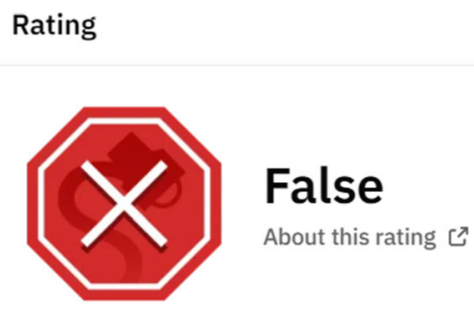

Fig. 1 Identification of event as rumor from snopes.com 
MENU $Q$

POLITIFACT

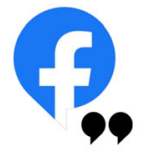

Facebook posts

stated on March 15, 2020 in a Facebook post:

"Bill Gates told us about the coronavirus in 2015."

FACEBOOK FACT-CHECKS

CORONAVIRUS

○ FACEBOOK POSTS

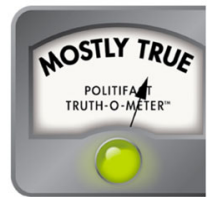

Fig. 2 Identification of event as non-rumor from Politifact.com

present in the tweet, such as can't to "cannot", don't to "do not". Finally, non-ASCII characters are removed and text is converted to lower string. The detailed function of text preprocessing is shown in Fig. 4. These clean tweets are used to pull out lexical features and word embedding. The content-based components are extracted from the original tweets.

Data encoding in the numeric form must be needed for the text data to input a deep neural network. Besides the approximate average length of all posts, we consider the maximum lengths for the sequence as 100 . The Tokenizer separates the post into different tokens and used as a word dictionary. This word dictionary is used to convert the message into a sequence of integers using the text_to_sequence function. Padding after the post is performed to make all sequences equal to maximum length. The embedding layer is used to understand the meaning of words in a post, transforming each word into an $\mathrm{n}$-dimensional word embedding vector by taking a sequence of posts as an input. The output of this embedding layer passed as an input to the BiLSTM model.

\subsection{Feature selection}

Text and time series data are the main attraction of recent work in rumor detection. But along with text, we have targeted features like a user, content-based, and lexical features from posts. User-based features include registration age, description length, follower count, etc. Lee and Bosch explored lexical and content-based features for identifying the variety in languages [41]. The content-based features are grammar-level features, including part of speech (POS)

Will Gargling with Salt Water Eliminate the COVID-19 Coronavirus?

Will Gargling with Salt Water or Vinegar 'Eliminate' the COVID-19 Coronavirus?
Will Gargling with Vinegar 'Eliminate' the COVID-19 Coronavirus?

Will Gargling with Salt Water or Vinegar 'Eliminate' the COVID-19 Coronavirus?

Gargling with Salt Water or Vinegar Eliminate COVID-19 Coronavirus?

Original News
Search Queries for Data Collection

Fig. 3 Example of search queries for data collection of events 
Table 3 Sample list for rumor events

\begin{tabular}{llll}
\hline $\begin{array}{l}\text { Sr. } \\
\text { no. }\end{array}$ & Date & Event story & Post_count \\
\hline 1 & $09-03-2020$ & $\begin{array}{c}\text { A homemade hand sanitizer made with Tito's Vodka can be used to fight the } \\
\text { new coronavirus. }\end{array}$ & 2186 \\
& & & 37 \\
2 & $11-03-2020$ & Will an Asteroid Hit Earth in April 2020? & 123 \\
3 & $21-03-2020$ & Says drinking a bleach solution will prevent you from getting the coronavirus & 996 \\
4 & $21-03-2020$ & Don't hold your breath. This isn't a credible way to test for coronavirus & 60 \\
5 & $23-03-2020$ & Will Eating Bananas Prevent Coronavirus Infection? & 125 \\
6 & $23-03-2020$ & Did Nostradamus Predict the COVID-19 Pandemic? & 143 \\
7 & $26-03-2020$ & Can You Get a Free Coronavirus Test by Donating Blood? & 144 \\
8 & $26-03-2020$ & Will Gargling with Salt Water or Vinegar 'Eliminate' the COVID-19 Coro- \\
& & navirus? & 17 \\
9 & $26-03-2020$ & Will Sipping Water Every 15 Minutes Prevent a Coronavirus Infection? & 110 \\
10 & $28-03-2020$ & Beware of rumors of robbers posing as COVID testers & 4971 \\
11 & $29-03-2020$ & Does 'Every Election Year' Have a Coinciding Disease? & \\
\hline
\end{tabular}

tagging, count of question marks, exclamatory marks, word count, etc. However, lexical features from each post are identified using the Empath open-source library [14] to analyze the text into lexical features, similar to Linguistic Inquiry and Word Count (LIWC). It provides a total of 190 lexical features. Table 6 shows the identified features from each category. We have extracted 8 features from the user category, 12 from content-based, and 190 from the lexical category. Principal component analysis (PCA) was applied as a dimension reduction technique on 190 features from the lexical category. The optimum number of components is determined using the cumulative explained variance graph in Fig. 5 and concluded with 125 as principal components. These features are normalized using standard scalar and feed to a multilayer perceptron (MLP), one of the deep neural networks.

Features are represented through boxplot and heatmap in feature selection from the user and content-based category. Figure 6 shows the boxplot for value distribution of user registration

Table 4 Sample list for non-rumor events

\begin{tabular}{|c|c|c|c|}
\hline $\begin{array}{l}\text { Sr. } \\
\text { no. }\end{array}$ & Date & Event story & Post_count \\
\hline 1 & 24-03-2020 & Was 'Coronavirus' Replaced with 'Chinese Virus' in Trump's Notes? & 10,304 \\
\hline 2 & $27-03-2020$ & Bill Gates told us about the coronavirus in 2015 & 5000 \\
\hline 3 & $27-03-2020$ & Was COVID-19 Discovered in the US and South Korea on the Same Day? & 52 \\
\hline 4 & $27-03-2020$ & Are Most Cruise Ships Registered Under Foreign Flags & 1474 \\
\hline 5 & $27-03-2020$ & $\begin{array}{l}\text { Did Video Show Italian Army Trucks Transporting Coffins Amid COVID-19 } \\
\text { Pandemic? }\end{array}$ & 92 \\
\hline 6 & $27-03-2020$ & Is This a Photo of an American Revolutionary War Vet? & 865 \\
\hline 7 & $28-03-2020$ & $\begin{array}{l}\text { Spectrum will provide free internet to students during coronavirus school } \\
\text { closures }\end{array}$ & 2850 \\
\hline 8 & $28-03-2020$ & Does 'Triscuit' Mean 'Electric Biscuit'? & 68 \\
\hline 9 & 01-04-2020 & $\begin{array}{l}\text { Did Empire State Building Display 'Siren’ Lights During COVID-19 Pan- } \\
\text { demic? }\end{array}$ & 9994 \\
\hline 10 & 01-04-2020 & Did Cities Close Schools, Businesses During the 1918 Pandemic? & 36 \\
\hline 11 & 01-04-2020 & Did the Trump Administration Send 18 Tons of PPE to China in Early $2020 ?$ & 1092 \\
\hline
\end{tabular}


Table 5 Details of real-world and benchmarked dataset

\begin{tabular}{lllll}
\hline Name & Real-World & \multicolumn{2}{l}{ Benchmarked } & \\
\cline { 4 - 5 } & English Only & Existed & Extracted & English Only \\
& 70 & 992 & 990 & 986 \\
Total Events & 51 & 498 & 498 & 498 \\
Total Rumor Events & 18 & 494 & 492 & 489 \\
Total Non-Rumor Events & 85,560 & 340,176 & 274,530 & 267,708 \\
Total Posts & 47,209 & 132,470 & 105,256 & 104,920 \\
Total Rumored Posts & 38,351 & 207,706 & 169,274 & 162,788 \\
Total Non-Rumored Posts & 2 & 10 & 2 & 2 \\
Minimum Posts Per Event & 10,304 & 3029 & 2838 & 2702 \\
Maximum Posts Per Event & & & & \\
\hline
\end{tabular}

age and it can be noticed that the user sending genuine posts is much older on Twitter than the user sending fake posts. Figure 7 shows the difference between the correlation matrix of features from user and content-based groups related to rumor and non-rumor posts. It can be observed that few features (retweet count and follower count, verified user and follower count) in non-rumor data are highly correlated.

\subsection{Proposed model}

This section presents the details of various deep learning models implemented in this research, including existing models such as words Embedding, BiLSTM and MLP, and newly proposed models, which are explained below:

\subsubsection{Word embedding}

The dataset contains several tweets $t w t$ and every tweet $t w t$ is encompassed of an order of $n$ words, i.e. $t_{1}, t_{2}, \ldots . t_{n}$. Each word $t_{i}$ transform into an embedding vector $w_{i} \in E^{m}$, called as word embedding. The Keras embedding layer is utilized in this research. The input to the embedding layer contains an input matrix of two dimensional, also known as word embedding matrix represented by $E^{l \times m}$, where $l$ is the tweet's length, and $\mathrm{m}$ is the dimension of word

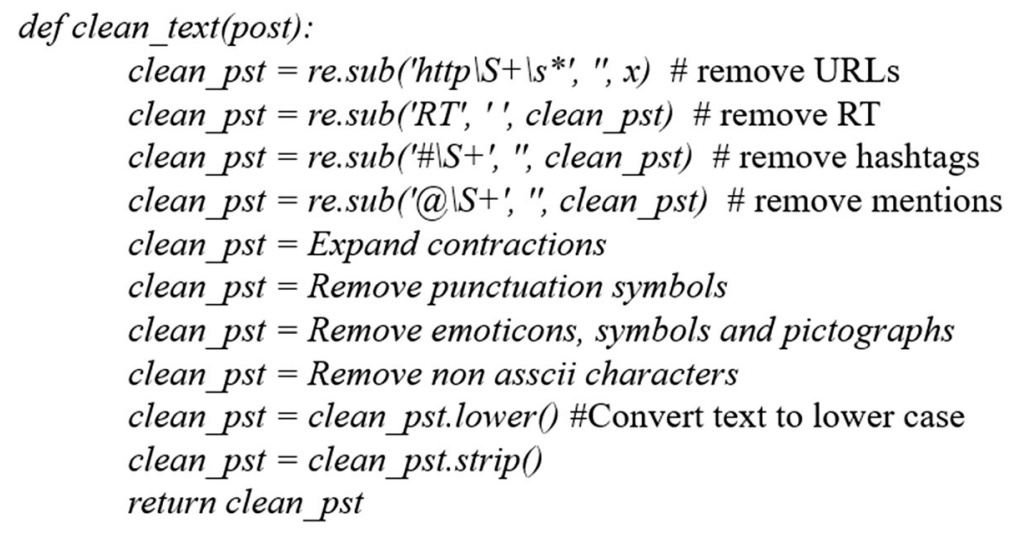

Fig. 4 Pseudocode for text preprocessing 
Table 6 Identified features from user, content and lexical category

\begin{tabular}{|c|c|}
\hline Category & Features \\
\hline User & $\begin{array}{l}\text { User_Registration_Age, Is_Verified_user?, User_Description_Length, Follower_count, } \\
\text { Friends_count, Favourite_count, Status_count, User_Location_present }\end{array}$ \\
\hline $\begin{array}{r}\text { Content } \\
\text { based }\end{array}$ & $\begin{array}{l}\text { \#Hashtags, \#URLs, \# Question_Marks, \#Exxlamatory, \#Mentions Retweet_count, Word_count, } \\
\text { Sentiment_score, Is_Media_present, Tweet_favorite_count, Tweet_geo_location_present, } \\
\text { Tweet_reply_count }\end{array}$ \\
\hline Lexical & $\begin{array}{l}\text { alcohol, ancient, anger, animal, anonymity, anticipation, appearance, art, attractive, banking, } \\
\text { beach, beauty, blue_collar_job, body, breaking, business, car, celebration, cheerfulness, } \\
\text { childish, children, cleaning, clothing, cold, college, communication, competing, computer, } \\
\text { confusion, contentment, cooking, crime, dance, death, deception, disappointment, disgust, } \\
\text { dispute, divine, domestic_work, dominant_heirarchical, dominant_personality, driving, eating, } \\
\text { economics, emotional, envy, exasperation, exercise, exotic, fabric, family, farming, fashion, } \\
\text { fear, feminine, fight, fire, friends, fun, furniture, gain, giving, government, hate, healing, health, } \\
\text { hearing, help, heroic, hiking, hipster, home, horror, hygiene, independence, injury, internet, } \\
\text { irritability, journalism, joy, kill, law, leader, legend, leisure, liquid, listen, love, lust, magic, } \\
\text { masculine, medical_emergency, medieval, meeting, messaging, military, money, monster, } \\
\text { morning, movement, music, musical, negative_emotion, neglect, negotiate, nervousness, night, } \\
\text { noise, occupation, ocean, office, optimism, order, pain, party, payment, pet, philosophy, phone, } \\
\text { plant, play, politeness, politics, poor, positive_emotion, power, pride, prison, programming, } \\
\text { rage, reading, real_estate, religion, restaurant, ridicule, royalty, rural, sadness, sailing, school, } \\
\text { science, sexual, shame, shape_and_size, ship, shopping, sleep, smell, social_media, sound, } \\
\text { speaking, sports, stealing, strength, suffering, superhero, surprise, swearing_terms, swimming, } \\
\text { sympathy, technology, terrorism, timidity, tool, torment, tourism, toy, traveling, trust, ugliness, } \\
\text { urban, vacation, valuable, vehicle, violence, war, warmth, water, weakness, wealthy, weapon, } \\
\text { weather, wedding, white_collar_job, work, worship, writing, youth, zest }\end{array}$ \\
\hline
\end{tabular}

embedding. The parameters used in the Embedding layer are the size of embedding dimension $(m)=32$, vocabulary size $=1000$ and length of the sequence $(l)=100$.

\subsubsection{Bidirectional LSTM (BiLSTM)}

We have used a variant of RNN as a bidirectional LSTM model (BiLSTM), which involves forward LSTM and backward LSTM. LSTM specifically fails to remember part of the historical data through three entryways (input door, forget door and output door), adds the

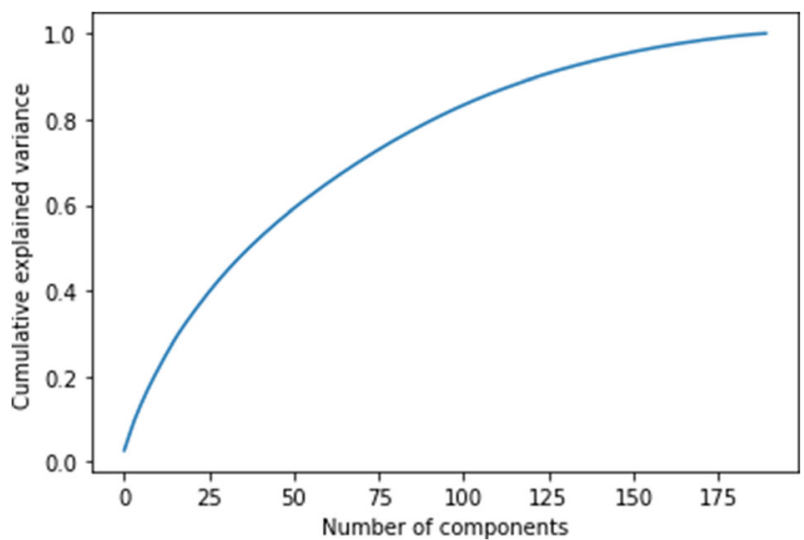

Fig. 5 Cumulative explained variance graph for PCA components 


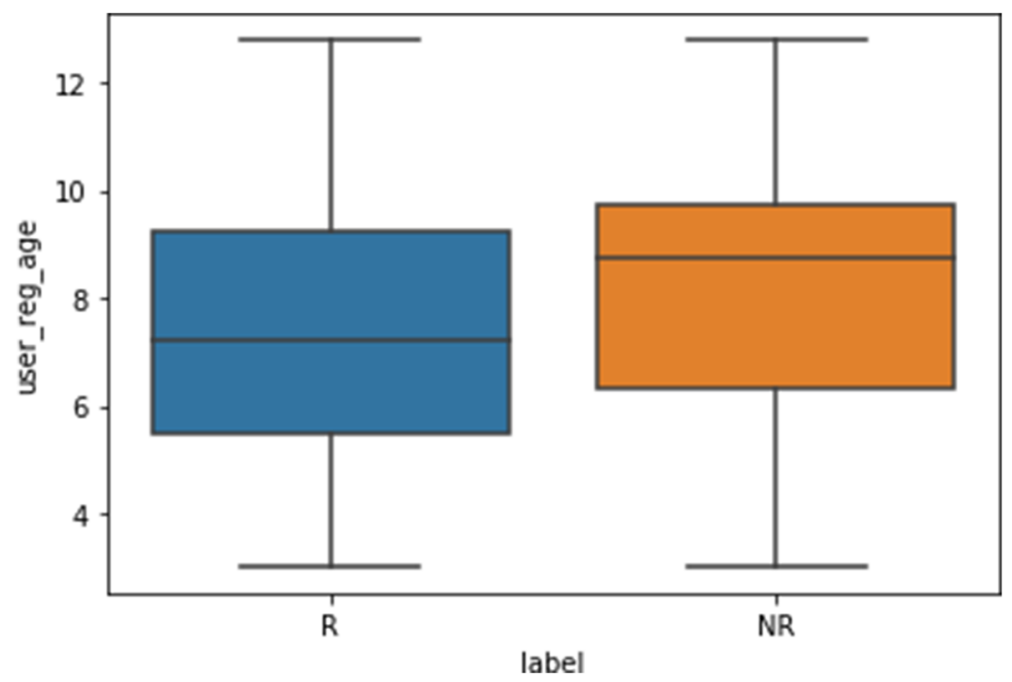

Fig. 6 Distribution of user registration age over rumor and non-rumor posts

amount of the current information data, and incorporates it into the present status to create the output state. The word embedding vector of posts is given as input to BiLSTM and represented by the BiLSTM_Embed model.

\subsubsection{Multilayer perceptron (MLP)}

MLP is one of the deep neural network (DNN) used to learn the post-wise features from the user, lexical and content-based features. A feature vector of 200 is given as input to MLP. A feature vector from MLP and BiLSTM is combined and provided as input to a densely connected layer in a hybrid model. lex_PCA and UCL_PCA models are executed explicitly on MLP.

\subsubsection{Overview of proposed models}

Based on the above-mentioned existing models, we have implemented BiLSTM_Embed, Lex_PCA, UCL_PCA and BiLSTM_UCL models.

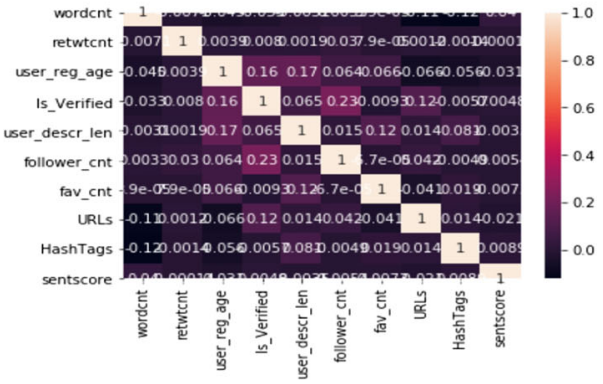

(a) Rumor

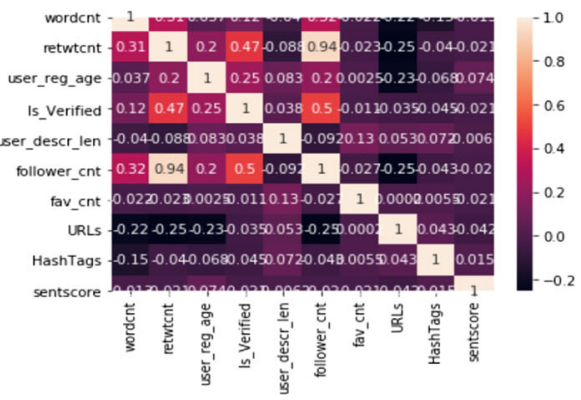

(b) Non-Rumor

Fig. 7 Correlation matrix for rumor and non-rumor posts 
- BiLSTM_Embed - Word embedding vector generated in the word embedding layer is feed into the BiLSTM model having 2 dense layers.

- Lex_PCA - 190 features from the lexical category are reduced to 125 using the PCA technique. To understand the significance of PCA, the Lex_PCA model is implemented and feeds with 125 principal components of lexical attributes as input. Lex_PCA model is implemented with 3 dense layers.

- UCL_PCA - model combines 20 features from user and content-based categories and 125 components from lexical category. Altogether, 145 features are normalized using standard scalar and passed as input to MLP.

- BiLSTM_UCL - Combines the features vector from BiLSTM_embed and UCL_PCA. Figure 8 shows the block diagram for the BiLSTM_UCL model's input and output.

The final proposed BiLSTM_UCL is a combination of BiLSTM_Embed and UCL_PCA, depicted as proposed deep learning architecture in Fig. 9. The variance of RNN and extension of basic LSTM as Bidirectional LSTM (BiLSTM) is used for the sequence of posts. BiLSTM processes the text forward and backward, providing additional context to the network with a better learning on the problem. After applying the post sequence to BiLSTM, we get a feature vector text_feat and feed as input to this model's fully connected layer $f_{c_{1}}$. We extracted different feature vectors for each post, such as user-based features as UserVect having user registration age, friend count, etc. Content-based features as ContVect have features like a count of Question Marks and the number of URLs, etc. and lexical features after applying PCA as LexVect. The features of each post are combined into a different vector such as FeatVect = $\left\{\right.$ UserVect, ContVect, LexVect\}\} and they are given as input to fully connected layer $\mathrm{fc}_{3}$ to get the second feature vector. The feature vectors from both the networks and dense layers $f c_{1}$ and $f c_{4}$ are merged and input to dense layer $f c_{5}$. The activation function used are relu in the

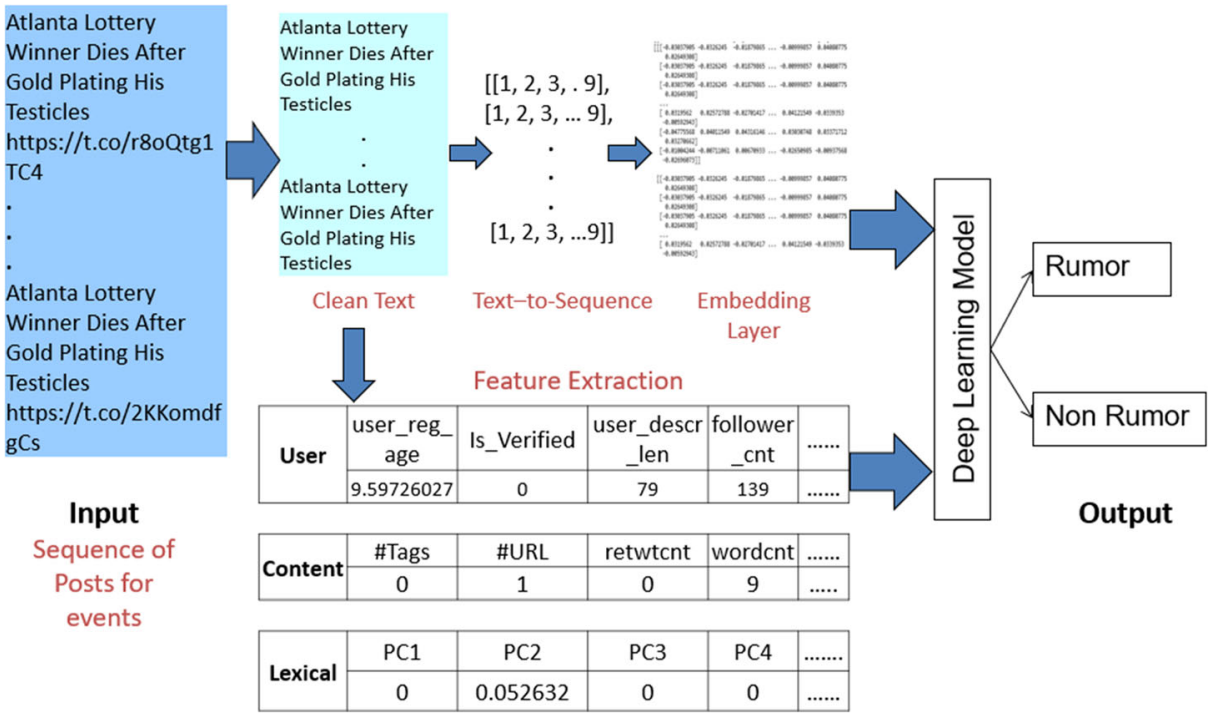

Fig. 8 Block diagram for input and output of BiLSTM_UCL model 


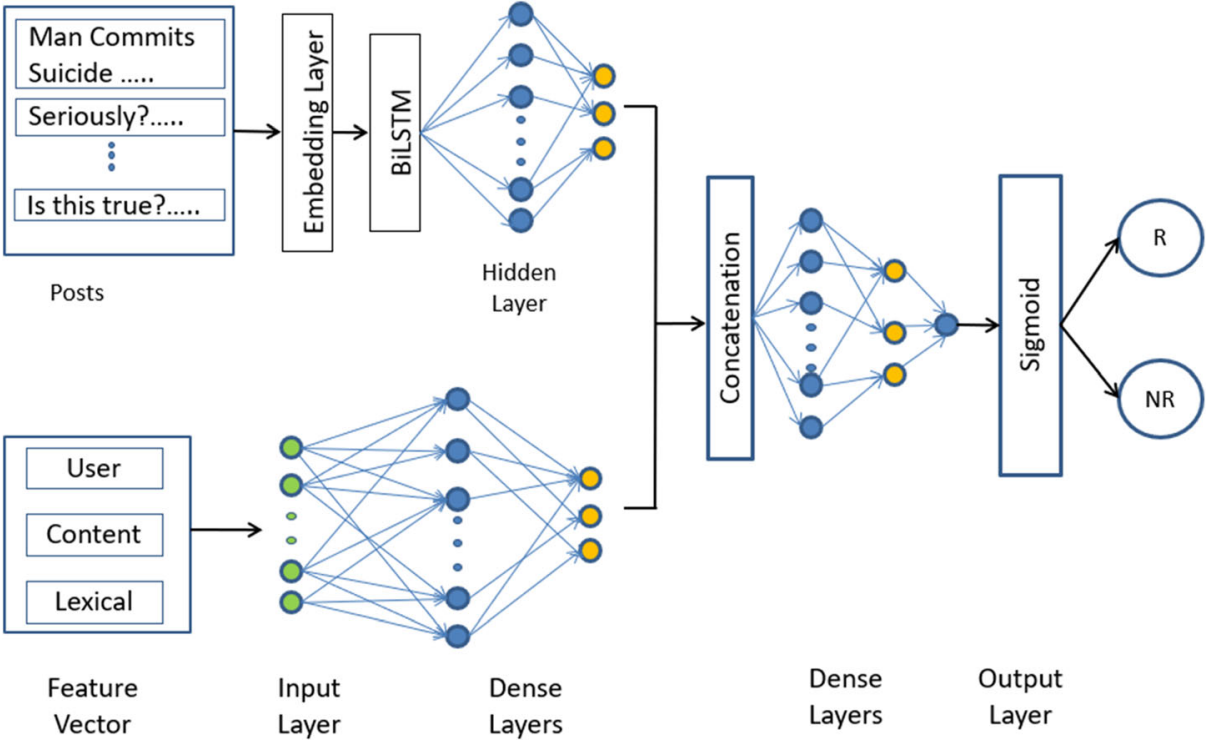

Fig. 9 Proposed architecture of BiLSTM_UCL model

dense layer and the sigmoid in the output layer. Figure 10 represents a summary of the BiLSTM_UCL model.

\section{Experimental results}

This section presents the dataset used, baseline approaches, experimental environment, evaluation metrics and experimental analysis on various deep learning models.

\subsection{Dataset}

The proposed method is evaluated on a real-world and benchmarked dataset of Twitter. Earlier, Table 7 explains the statistics of collected data and benchmarked datasets. The actual data collected from Twitter is significantly less as compared to the benchmarked dataset. Therefore, we have combined benchmarked and real-world data to get an extended dataset. Table 7 shows the details of benchmarked and extended real-world datasets. Thus, original benchmarked data get grown event-wise by $7 \%$ and post-wise by $32 \%$. Table 7 shows the actual data size used in the experimental evaluation. Data availability after extraction varies in different papers because few posts are not available at the time of data extraction from Twitter. We have split the dataset as $70 \%$ for training, $20 \%$ for testing and $10 \%$ for validation.

\subsection{Baseline approaches}

Following baseline, algorithms are identified to compare and evaluate the proposed deep learning models. 


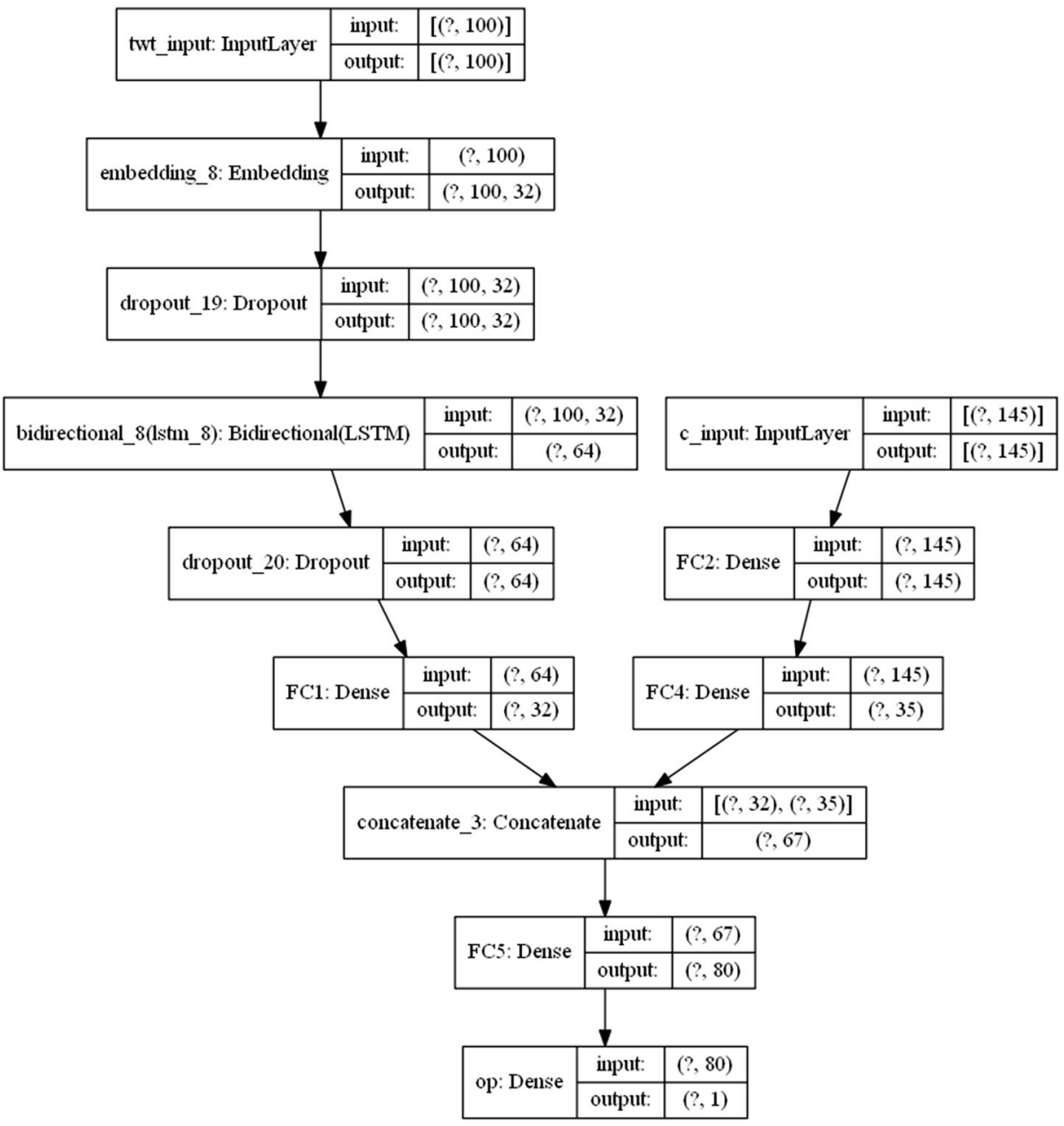

Fig. 10 BiLSTM_UCL model summary

GRU-II [25] experimented with different variants of RNN on time-series posts and revealed that GRU with 2-layers presents good performance.

Table 7 Details of benchmarked and extended real-world dataset

\begin{tabular}{lll}
\hline Name & Benchmarked & Real-world-extended \\
\hline Total Events & 986 & 1056 \\
Total Rumor Events & 498 & 549 \\
Total Non-Rumor Events & 489 & 507 \\
Total Posts & 267,708 & 353,268 \\
Total Rumored Posts & 104,920 & 152,129 \\
Total Non-Rumored Posts & 162,788 & 201,139 \\
Minimum Posts Per Event & 2 & 2 \\
Maximum Posts Per Event & 2702 & 10,304 \\
\hline
\end{tabular}


HAS-BLSTM [17] used a hierarchical attention model for social information with three hierarchy levels as word, post and subevent and utilized the BiLSTM model for rumor detection.

CAMI [42] discover that the CNN model can extract the significant features from post sequence and such models are suitable for early detection of rumors.

CSI [27] proposed a model for fake news classification using features based on the text of an article, the temporal characteristics of user reply and origin users broadcasting it. They combined the RNN model with a deep neural network (DNN) by integrating features from all three categories to get a more accurate rumor classification.

\subsection{Experimental setup and evaluation metrics}

The environmental setup used for implementation includes a scientific python development environment as Spyder-anaconda, tweepy library to access Twitter data and Keras with Tensorflow for deep learning. For the GPU environment, we have used the Google Colab cloud service. We have used tweepy API for collecting real-world data from Twitter.

For evaluation metrics, we adopted Accuracy, Precision, Recall and F1 scores for a comprehensive evaluation are defined in Eqs. (1), (2), (3), (4). The confusion matrix summarizes predicated results over actual results, as shown in Fig. 11, where R stands for rumor and NR for nonrumor. Accuracy is a fraction of correct predictions overall predictions. The quantity of accurate positive results divided by the quantity of positive results predicted by the classifier is called Precision. The recall is the quantity of correct positive results divided by the amount of all relevant samples.

$$
\text { Accuracy }=\frac{T P+T N}{T P+T N+F P+F N}
$$

$$
\text { Precision }=\frac{T P}{T P+F P}
$$

\begin{tabular}{|c|c|c|c|c|}
\hline & & & \multicolumn{2}{|c|}{ Predicted Class } \\
\hline & & & Positive (R) & Negative(NR) \\
\hline \multirow{2}{*}{$\frac{\frac{y}{\tilde{y}}}{\frac{d}{v}}$} & $\begin{array}{l}0 \\
:=0 \\
0 \\
0 \\
0 \\
0\end{array}$ & $\widehat{\approx}$ & $\begin{array}{c}\text { True Positive } \\
\text { (TP) }\end{array}$ & $\begin{array}{c}\text { False Negative } \\
\text { (FN) }\end{array}$ \\
\hline & 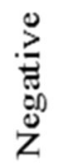 & 正 & $\begin{array}{c}\text { False Positive } \\
\text { (FP) }\end{array}$ & $\begin{array}{c}\text { True Negative } \\
\text { (TN) }\end{array}$ \\
\hline
\end{tabular}

Fig. 11 Confusion matrix for rumor detection 


$$
\begin{gathered}
\text { Recall }=\frac{T P}{(T P+F N)} \\
F 1=\frac{2 * \text { Recall } * \text { Precision }}{\text { Recall }+ \text { Precision }}
\end{gathered}
$$

\subsection{Experimental analysis}

This section explains the optimal hyperparameters used to set up different models in the research and experimental analysis. Table 8 shows the optimal hyperparameters used in the experiment, which involves the parameters used in various deep learning models, activation function, loss function.

The performance of various models is evaluated to finalize the proposed hybrid model. We have used binary_crossentropy as the loss function and Adagrad as the optimizer. The models are trained with a batch size of 32. However, the hyperparameters used for Adagrad are learning rate as 1e-1 and epsilon as 1e-07. Early stopping with the patience of 3 and drop out of 0.5 is used to avoid the overfitting of the model. Due to the early stopping number of epochs varies from 10 to 50, whereas the models are evaluated for 100 epochs. The accuracy of a model is verified with the learning curve of accuracy and loss to training and validation data. Figure 12 shows the learning curve of accuracy and loss for the BiLSTM_UCL model.

Initially, PCA applied on lexical features and given as input of 125 principal components to MLP called as Lex_PCA model shows the accuracy of 91\%. UCL_PCA is an MLP model that takes 145 features as an input, where 12 features from content-based, 8 from the user group and 125 features from the lexical category after applying PCA. The model trained with UCL features where UCL stands for User-Content-Lexical, which shows an accuracy of $93 \%$. The BiLSTM model considers posts with word embedding as input to the bidirectional LSTM model and offers 95\% accuracy. This model has 5 dense layers in MLP. The third model, BiLSTM_USL, combines the output of the previous two models and shows an accuracy of $97 \%$.

Table 9 compares experimental results on various deep learning models for real-world and benchmarked datasets. Here, the actual data collected from Twitter is significantly less than the

Table 8 Details of optimal hyperparameters

\begin{tabular}{ll}
\hline Parameter name & Value of parameter \\
\hline Vocabulary size & 1000 \\
Sequence length & 100 \\
Dropout & 0.5 \\
Adagrad Learning rate & 0.001 \\
Epoch & 100 \\
Number of Dense layers & 5 \\
Batch Size & 32 \\
Loss function & binary_crossentropy \\
Activation Function & Relu \\
Activation Function in Output Layer & Sigmoid \\
Loss Function & Adagrad \\
\hline
\end{tabular}



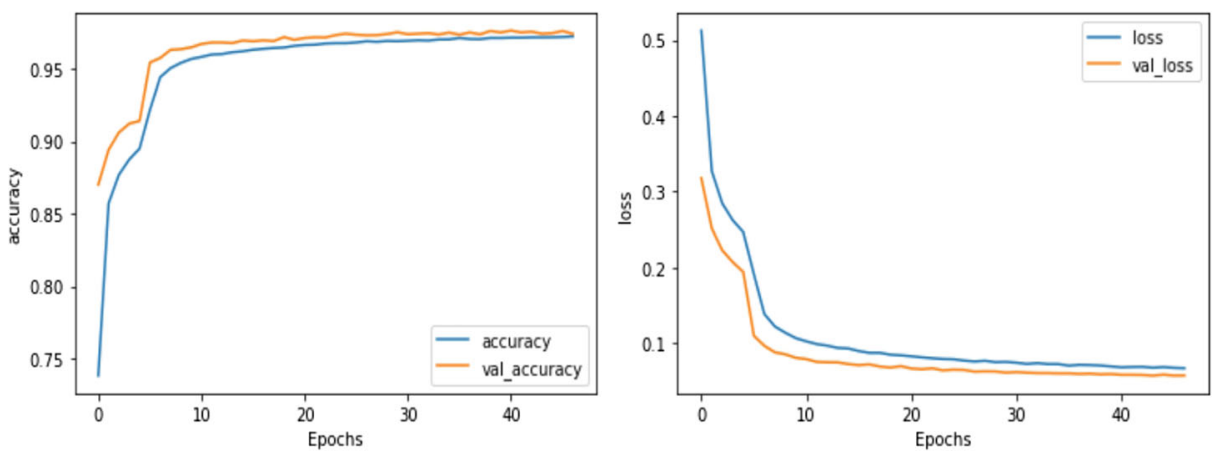

Fig. 12 The learning curve of accuracy and loss for the BiLSTM_UCL model

benchmarked dataset. Therefore the proposed method is evaluated on the extended dataset. Table 9 expresses a significant improvement in precision and recall value from 0.90 to 0.96 for rumor events. Also, it can be observed that results are slightly similar on a benchmarked and extended dataset.

From Table 10, it can be observed that combining BiLSTM_Embed and UCL_PCA model improves the accuracy, which shows the accuracy of $97 \%$ for the BiLSTM_UCL model. CSI [27] shows the highest accuracy of $89 \%$ from the previous work, which the Lex_PCA model shows. The experimental results shown in Table 10 are the values taken from the results mentioned in the related research paper for similar datasets and methodology. The experimental results of the proposed method are tested on a benchmarked dataset. Figure 13 presents the comparison of the proposed model with existing models concerning accuracy. Figure 14 shows the improvement in all implemented models where accuracy improves from $89 \%$ to 97\%. The results from overall experiments conclude that the proposed BiLSTM_UCL model shows an excellent enhancement in the accuracy of rumor detection.

\subsection{Discussion}

Table 9 presents the performance of proposed models on two datasets of different sizes and shows similar performance in terms of precision, recall and F1 score. Deep learning models are most suitable on large dataset and experiments in this research demonstrates that proposed method is scalable.

Table 9 Performance of proposed models on benchmarked and real-world dataset

\begin{tabular}{|c|c|c|c|c|c|c|c|c|}
\hline \multirow[t]{2}{*}{ Sr. no. } & \multirow[t]{2}{*}{ Models } & \multirow[t]{2}{*}{ Class } & \multicolumn{3}{|c|}{ Benchmarked dataset } & \multicolumn{3}{|c|}{ Real-world extended dataset } \\
\hline & & & Precision & Recall & $\mathrm{F} 1$ & Precision & Recall & $\mathrm{F} 1$ \\
\hline \multirow[t]{2}{*}{1.} & \multirow[t]{2}{*}{ Lex_PCA } & NR & 0.88 & 0.94 & 0.91 & 0.91 & 0.94 & 0.92 \\
\hline & & $\mathrm{R}$ & 0.90 & 0.80 & 0.85 & 0.91 & 0.87 & 0.89 \\
\hline \multirow[t]{2}{*}{2.} & \multirow[t]{2}{*}{ UCL_PCA } & NR & 0.92 & 0.97 & 0.95 & 0.92 & 0.97 & 0.95 \\
\hline & & $\mathrm{R}$ & 0.95 & 0.87 & 0.91 & 0.96 & 0.89 & 0.93 \\
\hline \multirow[t]{2}{*}{3.} & \multirow[t]{2}{*}{ BiLSTM_Embed } & NR & 0.95 & 0.97 & 0.96 & 0.96 & 0.96 & 0.96 \\
\hline & & $\mathrm{R}$ & 0.95 & 0.93 & 0.94 & 0.95 & 0.94 & 0.95 \\
\hline \multirow[t]{2}{*}{4.} & \multirow[t]{2}{*}{ BiLSTM_UCL } & NR & 0.97 & 0.97 & 0.98 & 0.97 & 0.98 & 0.98 \\
\hline & & $\mathrm{R}$ & 0.96 & 0.98 & 0.97 & 0.96 & 0.97 & 0.97 \\
\hline
\end{tabular}


Table 10 Experimental results

\begin{tabular}{|c|c|c|c|c|c|c|}
\hline Sr. no. & Models & Class & Accuracy & Precision & Recall & $\mathrm{F} 1$ \\
\hline \multirow[t]{2}{*}{1} & \multirow[t]{2}{*}{ GRU-II [25] } & $\mathrm{R}$ & \multirow[t]{2}{*}{0.881} & 0.851 & 0.95 & 0.898 \\
\hline & & NR & & 0.93 & 0.8 & 0.86 \\
\hline \multirow[t]{2}{*}{2} & \multirow[t]{2}{*}{ HSA-BLSTM [17] } & $\mathrm{R}$ & \multirow[t]{2}{*}{0.844} & 0.87 & 0.67 & 0.757 \\
\hline & & NR & & 0.73 & 0.899 & 0.805 \\
\hline \multirow[t]{2}{*}{3} & \multirow[t]{2}{*}{ CAMI [42] } & $\mathrm{R}$ & \multirow[t]{2}{*}{0.777} & 0.744 & 0.848 & 0.793 \\
\hline & & NR & & 0.82 & 0.705 & 0.758 \\
\hline 4 & CSI [27] & - & 0.892 & - & - & 0.83 \\
\hline \multirow[t]{2}{*}{5} & \multirow[t]{2}{*}{ Lex_PCA } & $\mathrm{R}$ & \multirow[t]{2}{*}{0.89} & 0.90 & 0.80 & 0.85 \\
\hline & & NR & & 0.88 & 0.94 & 0.91 \\
\hline \multirow[t]{2}{*}{6} & \multirow[t]{2}{*}{ UCL_PCA } & $\mathrm{R}$ & \multirow[t]{2}{*}{0.93} & 0.95 & 0.87 & 0.91 \\
\hline & & NR & & 0.92 & 0.97 & 0.95 \\
\hline \multirow[t]{2}{*}{7} & \multirow[t]{2}{*}{ BiLSTM_Embed } & $\mathrm{R}$ & \multirow[t]{2}{*}{0.95} & 0.95 & 0.93 & 0.94 \\
\hline & & NR & & 0.95 & 0.97 & 0.96 \\
\hline \multirow[t]{2}{*}{8} & \multirow[t]{2}{*}{ BiLSTM_UCL } & $\mathrm{R}$ & \multirow[t]{2}{*}{0.97} & 0.96 & 0.98 & 0.97 \\
\hline & & NR & & 0.97 & 0.97 & 0.98 \\
\hline
\end{tabular}

The computational complexity of NN models are analyzed in terms of a multiplication per recovered output by Freire et al. in [15]. Parameters considered for MLP are [batch, $\mathrm{n}_{\mathrm{s}}, \mathrm{n}_{\mathrm{i}}$ ], where the batch is the batch size, $n_{s}$ is memory size and is $n_{i}$ features count. Considering the Lex_PCA, an MLP based model having 125 features and 3 dense layers with the number of neurons in each dense layer as $n d_{1}, n d_{2}$ and $n d_{3}$, then the computational complexity (CC) of the MLP model can be given in Eq. (5) as:

$$
C C_{M L P}=\underbrace{n_{s} n_{i} n d_{1}}_{a}+\underbrace{n d_{1} n d_{2}+n d_{2} n d_{3}}_{b}+\underbrace{n d_{3} n_{o}}_{c}
$$

Where $\mathrm{a}, \mathrm{b}$ and $\mathrm{c}$ represent contributions from the input, hidden and an output layer of MLP.

The limitation of this research is the real-world data collected is relatively less; therefore, we have extended the benchmarked dataset by combining collected realworld data with the existing dataset. Although the results are evaluated on the benchmarked dataset and baseline algorithms, the dataset is not entirely available due to Twitter's policy. In the previous work, methods are evaluated on Sina Weibo and Twitter dataset. However, this research assessed only the Twitter dataset and focused on only English posts.

\section{Conclusions}

The diffusion of rumors and their impact on society is a massive problem in current social networks. To combat this, we have come up with rumor detection using post-wise essential features. Compared to the previous work, where more importance was given to text and temporal features and showed a moderate accuracy, this paper focused on text, user, contentbased, and lexical category features. The BiLSTM with word embedding and MLP model with various features improves the accuracy. The experimental results compared with the state-ofthe-art approaches and show a good improvement in the accuracy. This research also fetched 


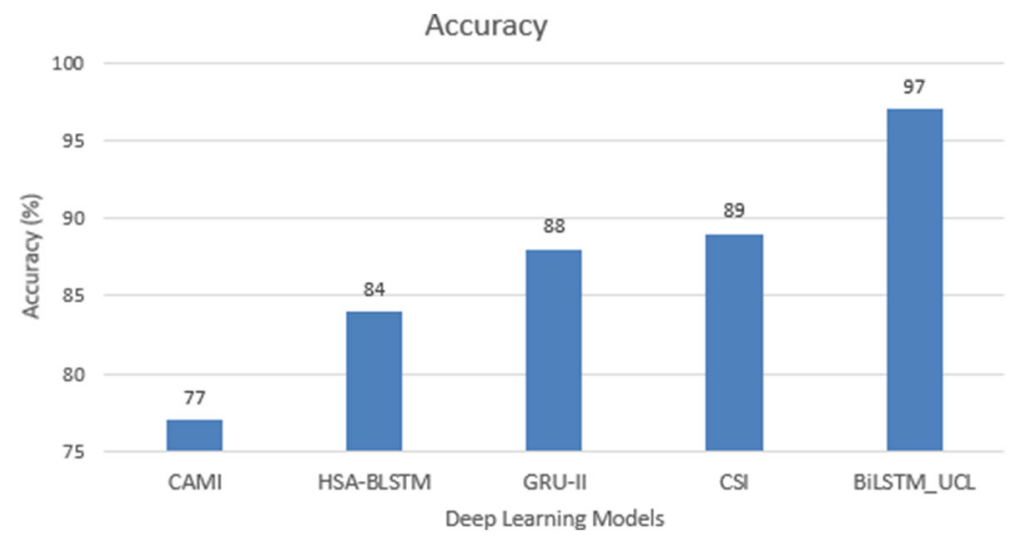

Fig. 13 Comparison of the proposed model with existing models

real-world data from Twitter and evaluated the experiment on both real-world and benchmarked datasets. Lexical features with PCA components show an accuracy of $89 \%$. The continuous improvements in the proposed models help finalize the combined model of BiLSTM_UCL with significant features from selected categories, demonstrating accuracy of $97 \%$.

In the future, we are planning to implement the same aspect with temporal features and attention models. The attention model can be utilized to identify the significant attributes from lexical features and will help to replace the feature selection method. In temporal characteristics, the word count of each post can be used to convert variable-length posts into fixed length posts. Also, future research may utilize multimedia-based features (such as image count, multimedia content present, is_real_image in the post? and video link present) to check the real news.

\section{Accuracy}

100

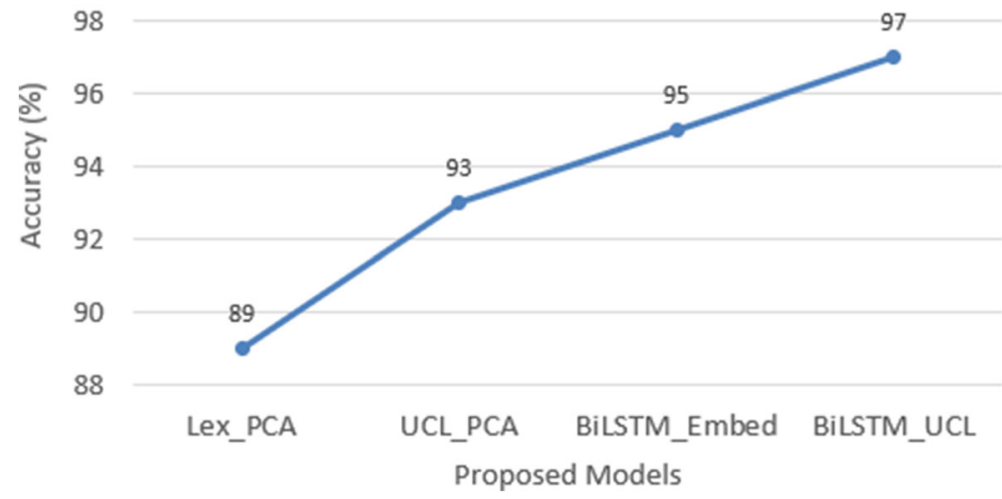

Fig. 14 Improvement in accuracy throughout the proposed models 


\section{Declarations}

Conflict of interest The research work presented here has not been submitted to, nor under review, at another journal or other publishing venue. All authors have participated in conception and design, analysis and interpretation of the data, drafting the article or revising it critically for important intellectual content, and approval of the final version.

\section{References}

1. After COVID-19 vaccine, blood or plasma donation not allowed. [Online]. Available: https://www. politifact.com/factchecks/2021/may/04/tiktok-posts/no-red-cross-isnt-warning-vaccinated-people-not-do/. Accessed 5 April 2021

2. Al-Sarem M, Boulila W, Al-Harby M, Qadir J, Alsaeedi A (2019) Deep learning-based rumor detection on microblogging platforms: a systematic review. IEEE Access 7:152788-152812

3. Asghar MZ, Habib A, Habib A, Khan A, Ali R, Khattak A (2019) Exploring deep neural networks for rumor detection. Journal of Ambient Intelligence and Humanized Computing:1-19

4. Bill Gates told us about the coronavirus in 2015 (n.d.) [Online]. Available: PolitiFact | Bill Gates warned in 2015 that we were unprepared for an infectious virus. Accessed 5 April 2021

5. Boididou C, Middleton SE, Jin Z, Papadopoulos S, Dang-Nguyen DT, Boato G, Kompatsiaris Y (2018) Verifying information with multimedia content on twitter: a comparative study of automated approaches. Multimed Tools Appl 77(12):15545-15571. https://doi.org/10.1007/s11042-017-5132-9

6. Bondielli A, Marcelloni F (2019) A survey on fake news and rumour detection techniques. Inf Sci 497:38-55

7. Castillo C, Mendoza M, Poblete B (2011) Information credibility on twitter. In: Proceedings of the 20th international conference on world wide web - WWW '11, p 675. https://doi.org/10.1145/1963405.1963500

8. Chen T, Li X, Yin H, Zhang J (2018) Call attention to rumors: deep attention based recurrent neural networks for early rumor detection. In: Pacific-Asia conference on knowledge discovery and data mining, pp 40-52

9. Chen W, Zhang Y, Yeo CK, Lau CT, Lee BS (2018) Unsupervised rumor detection based on users' behaviors using neural networks. Pattern Recogn Lett 105:226-233. https://doi.org/10.1016/j.patrec.2017. 10.014

10. COVID-19 killed fewer people than the flu. [Online]. Available: https://www.politifact.com/factchecks/ 2021/apr/27/facebook-posts/no-covid-19-hasnt-killed-fewer-people-flu/. Accessed 5 April 2021

11. COVID-19 Vaccine Cause Herpes (2021) [Online]. Available: https://www.snopes.com/fact-check/covid19-vaccine-herpes/. Accessed 5 April 2021

12. Facebook Social Network (n.d.) [Online]. Available: https://facebook.com

13. Fact Checking website (n.d.) [Online]. Available: https://www.factcheck.org/

14. Fast E, Chen B, Bernstein MS (2016) Empath: understanding topic signals in large-scale text. In: Proceedings of the $2016 \mathrm{CHI}$ conference on human factors in computing systems, pp 4647-4657

15. Freiredecarvalhosouza PJ, Osadchuk Y, Spinnler B, Napoli A, Schairer W, da Costa NMS, Prilepsky J, Turitsyn SK (2021) Performance versus complexity study of neural network equalizers in coherent optical systems. J Lightwave Technol 39:6085-6096

16. Gargling with salt water or Vinegar 'eliminate' the COVID-19 coronavirus from the throat (n.d.) [Online]. Available: Will Gargling with Salt Water or Vinegar 'Eliminate' the COVID-19 Coronavirus?|Snopes.com

17. Guo H, Cao J, Zhang Y, Guo J, Li J (2018) Rumor detection with hierarchical social attention network. In: Proceedings of the 27th ACM international conference on information and knowledge management, pp 943-951

18. Jogalekar NS, Attar V, Palshikar GK (2020) Rumor detection on social networks: a sociological approach. In: 2020 IEEE international conference on big data (big data), pp 3877-3884. https://doi.org/10.1109/ BigData50022.2020.9378149

19. Kaliyar RK, Goswami A, Narang P (2021) FakeBERT: fake news detection in social media with a BERTbased deep learning approach. Multimed Tools Appl 80(8):11765-11788

20. Kotteti CMM, Dong X, Qian L (2020) Ensemble deep learning on time-series representation of tweets for rumor detection in social media. Appl Sci 10(21):7541

21. Kumar S, Asthana R, Upadhyay S, Upreti N, Akbar M (2019) Fake news detection using deep learning models: A novel approach. Transactions on Emerging Telecommunications Technologies:e3767

22. Li X, Lu P, Hu L, Wang X, Lu L (2021) A novel self-learning semi-supervised deep learning network to detect fake news on social media. Multimedia Tools and Applications:1-9 
23. Lin X, Liao X, Xu T, Pian W, Wong K-F (2019) Rumor detection with hierarchical recurrent convolutional neural network. In: CCF international conference on natural language processing and Chinese computing, pp 338-348

24. Liu Y, Wu YB (2018) Early detection of fake news on social media through propagation path classification with recurrent and convolutional networks. AAAI

25. Ma J, Gao W, Mitra P, Kwon S, Jansen BJ (2016) Detecting rumors from microblogs with recurrent neural networks detecting rumors from microblogs with recurrent neural networks. In: Proceedings of the 25 th international joint conference on artificial intelligence (IJCAI 2016), July, pp 3818-3824

26. Ma J, Gao W, Wong K-F (2019) Detect rumors on twitter by promoting information campaigns with generative adversarial learning. In: The World Wide Web Conference, pp 3049-3055

27. Ruchansky N, Seo S, Liu Y (2017) Csi: a hybrid deep model for fake news detection. In: Proceedings of the 2017 ACM on conference on information and knowledge management, pp 797-806

28. Rumor Detection Public Dataset (n.d.) [Online]. Available-http://alt.qcri.org/ «wwgao/data/rumdect.zip

29. Shao C, Ciampaglia GL, Varol O, Yang K-C, Flammini A, Menczer F (2018) The spread of low-credibility content by social bots. Nat Commun 9(1):1-9

30. Sharaff A, Nagwani NK (2020) ML-EC2: an algorithm for multi-label email classification using clustering. International Journal of Web-Based Learning and Teaching Technologies (IJWLTT) 15(2):19-33

31. Sharaff A, Nagwani NK, Dhadse A (2016) Comparative study of classification algorithms for spam email detection. In: Emerging research in computing, information, communication and applications. Springer, pp 237-244

32. Sharaff A, Khurana S, Cheepurupalli K, Sahu T (2020) Personalized recommendation system with user interaction based on LMF and popularity model. In: 2020 international conference on system, computation, automation and networking (ICSCAN), pp 1-6

33. Shelke S, Attar V (2019) Source detection of rumor in social network - a review. Online Social Networks and Media 9:30-42. https://doi.org/10.1016/J.OSNEM.2018.12.001

34. Shelke S, Attar V (2020) Origin identification of a rumor in social network. In: Cybernetics, cognition and machine learning applications. Springer, pp 89-96

35. Shu K, Mahudeswaran D, Wang S, Lee D, Liu H (2020) Fakenewsnet: a data repository with news content, social context, and spatiotemporal information for studying fake news on social media. Big Data 8(3):171-188

36. Social network statistics. [Online]. Available: https://backlinko.com/social-media-users. Accessed 5 April 2021

37. Song C, Yang C, Chen H, Tu C, Liu Z, Sun M (2019) CED: credible early detection of social media rumors. IEEE Trans Knowl Data Eng

38. Srinivasarao U, Sharaff A (2021) Email sentiment classification using lexicon-based opinion labeling. In: Intelligent computing and communication systems. Springer, pp 211-218

39. Tchakounté F, Calvin KA, Ari AAA, Mbogne DJF (2020) A smart contract logic to reduce hoax propagation across social media. Journal of King Saud University-Computer and Information Sciences

40. Twitter Social Network (n.d.) [Online]. Available: https://twitter.com

41. van der Lee C, van den Bosch A (2017) Exploring lexical and syntactic features for language variety identification. Proceedings of the Fourth Workshop on NLP for Similar Languages, Varieties and Dialects (VarDial), pp 190-199

42. Yu F, Liu Q, Wu S, Wang L, Tan T (2017) A convolutional approach for misinformation identification. In: Proceedings of the 26th international joint conference on artificial intelligence, pp 3901-3907

43. Zubiaga A, Aker A, Bontcheva K, Liakata M, Procter R (2018) Detection and resolution of rumours in social media: a survey. ACM Computing Surveys (CSUR) 51(2):32

Publisher's note Springer Nature remains neutral with regard to jurisdictional claims in published maps and institutional affiliations. 